Article

\title{
Multidisciplinary Care of Patients with Facial Palsy: Treatment of 1220 Patients in a German Facial Nerve Center
}

\author{
Jonathan Steinhäuser ${ }^{1}$, Gerd Fabian Volk ${ }^{1,2,3}{ }^{\mathbb{D}}$, Jovanna Thielker ${ }^{1,2}$, Maren Geitner ${ }^{1,2}$, \\ Anna-Maria Kuttenreich ${ }^{1,2}$, Carsten M. Klingner ${ }^{2,3,4}{ }^{\mathbb{D}}$, Christian Dobel ${ }^{1,2}$ and Orlando Guntinas-Lichius ${ }^{1,2,3, * \mathbb{D}}$
}

1 Department of Otorhinolaryngology, Jena University Hospital, 07747 Jena, Germany; jonathan.steinhaeuser@live.de (J.S.); fabian.volk@med.uni-jena.de (G.F.V.); jovanna.thielker@med.uni-jena.de (J.T.); maren.geitner@med.uni-jena.de (M.G.); anna-maria.kuttenreich@med.uni-jena.de (A.-M.K.); christian.dobel@med.uni-jena.de (C.D.)

2 Facial Nerve Center Jena, Jena University Hospital, 07747 Jena, Germany; carsten.klingner@med.uni-jena.de 3 Center for Rare Diseases, Jena University Hospital, 07747 Jena, Germany

4 Department of Neurology, Jena University Hospital, 07747 Jena, Germany

* Correspondence: orlando.guntinas@med.uni-jena.de; Tel.: +49-3641-9329301; Fax: +49-3641-9329302

\section{check for}

updates

Citation: Steinhäuser, J.; Volk, G.F.; Thielker, J.; Geitner, M.; Kuttenreich, A.-M.; Klingner, C.M.; Dobel, C.; Guntinas-Lichius, O. Multidisciplinary Care of Patients with Facial Palsy: Treatment of 1220 Patients in a German Facial Nerve Center. J. Clin. Med. 2022, 11, 427. https://doi.org/10.3390/ jcm11020427

Academic Editor: Tetsuro Tamaki

Received: 29 November 2021

Accepted: 12 January 2022

Published: 14 January 2022

Publisher's Note: MDPI stays neutral with regard to jurisdictional claims in published maps and institutional affiliations.

Copyright: (C) 2022 by the authors. Licensee MDPI, Basel, Switzerland. This article is an open access article distributed under the terms and conditions of the Creative Commons Attribution (CC BY) license (https:// creativecommons.org/licenses/by/ $4.0 /)$.

\begin{abstract}
To determine treatment and outcome in a tertiary multidisciplinary facial nerve center, a retrospective observational study was performed of all patients referred between 2007 and 2018 . Facial grading with the Stennert index, the Facial Clinimetric Evaluation (FaCE) scale, and the Facial Disability Index (FDI) were used for outcome evaluation; 1220 patients (58.4\% female, median age: 50 years; chronic palsy: $42.8 \%$ ) were included. Patients with acute and chronic facial palsy were treated in the center for a median of 3.6 months and 10.8 months, respectively. Dominant treatment in the acute phase was glucocorticoids \pm acyclovir (47.2\%), followed by a significant improvement of all outcome measures $(p<0.001)$. Facial EMG biofeedback training $(21.3 \%)$ and botulinum toxin injections $(11 \%)$ dominated the treatment in the chronic phase, all leading to highly significant improvements according to facial grading, FDI, and FaCE $(p<0.001)$. Upper eyelid weight $(3.8 \%)$ and hypoglossal-facial-nerve jump suture $(2.5 \%)$ were the leading surgical methods, followed by improvement of facial motor function $(p<0.001)$ and facial-specific quality of life (FDI, FaCE; $p<0.05$ ). A standardized multidisciplinary team approach in a facial nerve center leads to improved facial and emotional function in patients with acute or chronic facial palsy.
\end{abstract}

Keywords: multidisciplinary care; Bell's palsy; chronic facial palsy; facial paralysis; facial nerve surgery; botulinum toxin; physiotherapy

\section{Introduction}

Peripheral facial palsy is the most frequent cranial nerve palsy causing significant functional and psychological morbidity. The management of patients with facial palsy can be challenging because there are over 50 etiologies [1]. The annual incidence of idiopathic Bell's palsy as the most frequent type of acute facial palsy is reported to be 20 to 40 of 100,000 persons [2]. As about $70 \%$ of the cases of acute facial palsy are Bell's palsy, the overall incidence of acute facial palsy is 29 to 57 of 100,000 persons per year [3]. Depending on the severity of the lesion, at least $30 \%$ of the cases will not recover completely. These cases will remain flaccid (chronic flaccid facial palsy) if not treated otherwise or develop post-paralytic synkinesis due to pathological facial nerve regeneration. There is a broad and continuously evolving spectrum of diagnostic and management approaches for facial palsy [4]. The management of patients with facial palsy often requires complex clinical decision-making [5]. Optimal diagnostics are needed to detect early the non-idiopathic cases, cases with worse prognosis, or candidates for immediate or early reconstruction surgery [1]. Although several clinical guidelines have been published, many cases still do 
not receive optimal treatment. Moreover, patients with a low probability of recovery are not referred or referred very late to specialized facial palsy services $[2,6]$.

The multidisciplinary centralized approach for facial palsy in a referral center has several advantages. Treatment in a specialized center allows a systematic and up-to-date assessment of facial function, functional and psychological considerations, assessment of quality of life, elaboration of guidelines, emotional support, comprehensive long-term care, and facilitated inclusion into clinical trials [5,7]. As such, a series of 3650 patients treated in Pittsburgh between 1963 and 1996 and a series of 1989 patients treated in Boston between 2003 and 2013, with a focus on decision-making but not on the outcome, have been published [4]. A multidisciplinary collaboration, including a wide variety of subspecialties, has proven effective for the treatment of patients with Bell's palsy [8]. A patient-centered approach, utilizing physiotherapy, targeted botulinum toxin injections, and selective surgical intervention offered by a multidisciplinary team can effectively reduce the burden of long-term disability for patients with Bell's palsy and longstanding sequelae [8]. The same has been shown for the management of facial paralysis following skull base surgery [5]. These patients profit from a multidisciplinary intervention because an individualized combination of pharmacologic therapy, physical therapy for facial neuromuscular retraining, and surgical intervention is needed for most patients [5]. Krane et al. reported on establishing a facial nerve center based on the experience with 22 surgical cases treated between 2014 and 2019 [9]. Of particular note is that not only standard facial grading but also facial-nerve-specific patient-related outcome measures (PROMs) were used to measure the outcome in a standardized manner. In doing so, Krane et al. could show that nerve transfer and free gracilis muscle transfer not only improved smile excursion and facial symmetry but also the quality of life of these patients [9]. The most recent report comes from the Sydney Facial Nerve Clinic on 145 patients treated between 2015 and 2018 [10]. The Sydney team also used both classical facial grading and PROMs for the initial assessment of the patients and during follow-up. This allowed them to show that it is not the physical level of function that a patient has but the social and psychological impact of their palsy that drives them to presentation in a specialized facial nerve center [10]. Another more historical but large ( $>1000$ patients) series focused on Bell's palsy [11-13]. These historical series do not include standardized outcome measures and are limited to the measurement of the physical dysfunction of the patients. These studies do not include data on the quality of life of the patients or their psychosocial dysfunction.

When assessing the outcome of a facial nerve center, it seems to be advisable to measure the outcomes with standardized grading tools and PROMs. This is of interest for patients with acute and chronic facial palsy. Therefore, we aim to report the experience from a German multidisciplinary facial nerve center, treating patients with acute and chronic facial palsy. The focus lies on the diagnostic and therapeutic management of 1220 patients. The patients are not only initially assessed and later on monitored by facial nerve grading but also from the beginning by facial nerve specific (PROMs). This allows us to critically analyze the outcome in our center. Furthermore, we can compare the results to other multidisciplinary centers using a similar approach.

\section{Material and Methods}

\subsection{Ethical Considerations}

The study was conducted according to the guidelines of the Declaration of Helsinki and approved by the Ethics Committee of the Jena University Jena, Germany (protocol code 4665-01/16; approved at 22 January 2016).

\subsection{Study Design and Inclusion Criteria}

This observational cohort study was based on the database of the Facial Nerve Center, Jena University Hospital, Jena, Germany. Standardized prospective data collection on all patients admitted with facial palsy was started in 2007. The patients were admitted to the Department of Otorhinolaryngology or the Department of Neurology of the Jena 
University Hospital, Jena, Germany. The database includes patients' baseline characteristics, all diagnostics, and data of all outpatient, daycare, and inpatient treatments. The multidisciplinary team approach was started in 2008. The team also includes a psychologist, speech therapists, and physiotherapists to see all new patients. The Facial Nerve Center in Jena was formally established in 2012. Here, we present all patients admitted with facial palsy (International Classification of Diseases [ID] codes G51.0, G51.1, G51.2, G51.8, and G51.9) between 2007 and 2018. The inclusion was limited to 2018 to allow an adequate follow-up. Only patients with hemifacial spasm (G51.3), facial myokymia (G51.4), and facial dystonia $\left(\mathrm{G} 24^{*}\right)$ were excluded. Otherwise, the study ought to reflect the complete health care service of the center for patients with any kind of facial palsy. Most patients came from Germany (99.3\%). Over the years, the number of referred patients has increased continuously, mainly from other federal states in Germany (Figure S1). The diagnostic and therapy approach was different for patients referred with acute facial palsy ( $\leq 90$ days after onset; acute palsy group) and patients with chronic facial palsy ( $>90$ days after onset; chronic palsy group) and depending on the underlying disease. In the chronic phase, patients were either referred with flaccid facial paralysis/flaccid paresis of parts of the face or post-paralytic facial synkinesis. Some patients were referred with acute or chronic flaccid paralysis, became patients with post-paralytic synkinesis after nerve repair, and were eventually also treated for synkinesis (Figure S2).

\subsection{Diagnostics and Assessment of Initial and Final Facial Nerve Function}

Depending on the etiology of acute facial palsy, or if unknown, a battery of diagnostics is performed. Basic diagnostics includes otorhinolaryngologic examination, including ultrasound of head and neck and a neurological examination, laboratory tests, pure tone audiometry, vestibular function tests, the stapedius reflex test, the gustatory test, and the Schirmer test $[14,15]$. Baseline electroneurography, blink reflex testing, and needle electromyography (EMG) were performed as fast as possible in case of acute facial palsy [16] If the first EMG was performed earlier than 14 days after onset, the examination was repeated at least once and later than 14 days after onset.

In the case of chronic facial palsy, EMG was also the central electrodiagnostic test to confirm complete denervation in chronic flaccid palsy or to confirm synkinetic reinnervation in the case of pathological reinnervation with post-paralytic synkinesis. Facial muscle ultrasound was introduced in 2011 and has been a routine diagnostic tool since 2013 in longterm flaccid facial palsy to analyze the viability of the facial muscles [17]. The most recently implemented diagnostic tool was diagnostic electrostimulation in 2015. This enables a direct electrophysiological evaluation of long-term denervated facial musculature [18,19].

At each presentation, the facial palsy is graded according to the Stennert index [20]. The Stennert index classifies the face at rest $(0-4$ points; $0=$ normal to $4=$ complete loss of resting tone) and during motion ( $0-6$ points; $0=$ normal to $6=$ no motion) separately. The points for both scores are summarized to a total score $(0=$ normal; $10=$ worst dysfunction $)$. Clinically, the palsy is defined as complete if the patient presents with a complete loss of motor function in the affected hemiface or if the palsy has deteriorated to a complete palsy during the inpatient course of treatment. Otherwise, the palsy is defined as incomplete palsy. The degree of facial nerve dysfunction is not classified as "paresis" = incomplete loss of facial nerve function and "paralysis" = complete loss of facial nerve function. Instead, we use here the umbrella terms "palsy" or "facial nerve dysfunction". The patient's perspective was regularly assessed using the German versions of two patient-reported outcome measures (PROMs). The Facial Clinimetric Evaluation (FaCE) scale and the Facial Disability Index (FDI) were used [21-23]. The FDI questionnaire comprises 10 Likert-type questions, divided into two domains, and includes physical function and social/wellbeing function. The physical function scale is scored from -25 (worst) to 100 (best). The social/well-being function scores range from 0 (worst) to 100 (best). Both FDI scales are summed to a total score. The FaCE has six independent domains: social function, facial movement, facial comfort, oral function, eye comfort, lacrimal control, and a total score 
incorporating all domains. Each FaCE score ranges from 0 (worst) to 100 (best). The 36-item SF-36 Health Survey (SF-36) measures the general quality of life [24]. Outcome criteria for all patients were the absolute change of the Stennert index and the PROMs between their first and last visits to the Facial Nerve Center.

The evaluation of patients with post-paralytic synkinesis included a psychological assessment of the suitability for in-house daycare EMG biofeedback training. This assessment contained the Body Dysmorphic Disorder Munich Module (BDD-MM) [25], the Beck Depression Inventory (BDI) [26], and the Liebowitz Social Anxiety Scale (LSAS) [27]. Results of this assessment were published recently and will not be presented here [28].

\subsection{Treatment}

In the case of acute facial palsy, the underlying disease directed the treatment under consideration of the German guideline for the treatment of facial palsy [29,30]. As symptomatic treatment and in each case of idiopathic facial palsy, a tapered course of corticosteroids over 7 days is regarded as standard treatment [30]. In the case of VZV reactivation, but also for many patients with idiopathic facial palsy, the patients received additional acyclovir for 5 days. Ceftriaxone was given for 7 days for patients with Lyme disease (mainly stage 2, early disseminated infection; seldom stage 3, late persistent Lyme disease).

The center offers a spectrum of surgical and non-surgical treatments, mainly used for patients with chronic palsy, published in detail elsewhere [31-33]. For this study, the surgical procedures were grouped into facial nerve reconstruction procedures, muscle transposition and static facial procedures, and eyelid surgery. The non-surgical treatments were categorized into physical therapy/speech therapy, electrotherapy, botulinum toxin injection, eye moisture chamber, eye drops, facial exercises at home, and in-house daycare facial biofeedback training.

\subsection{Statistics}

Statistical analyses were performed using IBM SPSS version 26.0 statistical software for Windows (Chicago, IL, USA). Maps to demonstrate the regional distributions of the referred patients were prepared with the software program PLZ-Diagramm 3.8 (Wessiepe, Grevenbroich, Germany). Differences between two independent subgroups for nominal data were compared with Pearson's chi-square test and between more than two subgroups with Fisher's exact test. Differences between two independent subgroups for metric data were compared with the $t$-test. Differences between two dependent subgroups for metric data were compared with the paired $t$-test and between more than two subgroups with univariate analysis of variance (ANOVA). A Bonferroni correction was applied in case of multiple comparisons in-between $>2$ subgroups. The probability of complete recovery from acute facial palsy was calculated by the Kaplan-Meier method. Recovery differences of the two subgroups were compared by the log-rank test. Multivariable analysis was performed using the Cox proportional hazards model to estimate the hazard ratio (HR) for recovery. For all statistical tests, significance was two-sided and set to $p<0.05$.

\section{Results}

\subsection{Baseline and Facial Palsy Characteristics}

Aggregate baseline data on the entire study group of 1220 patients $(58.4 \%$ female, median age: 50 years) are presented in Table S1. The characteristics showed differences between patients referred with acute versus chronic facial palsy (Table 1). The patients with acute palsy were referred at a median time of 1 day (range: $0-90) ; 76.2 \%$ of the patients with acute palsy were referred within $72 \mathrm{~h}$ after onset. The median time for patients with idiopathic etiology was 2 days (range: $0-90) ; 72.1 \%$ of the patients with idiopathic etiology were referred within $72 \mathrm{~h}$. The patients with chronic palsy were referred at a median time of 1.5 years (range: $0.2-70$ ). The proportion of female patients in the chronic palsy group was higher than in the acute palsy group $(p<0.0001)$. The rate of recurrent palsies was higher in the acute palsy group $(p=0.039)$. Patients with tumors as underlying etiology 
and congenital cases presented more frequently as chronic cases $(p<0.0001)$. The patients with acute palsy were older than chronic palsy cases $(p<0.0001)$.

Table 1. Comparison of patients' characteristics referred as a case of acute facial palsy ( $\leq 90$ days after onset) versus as chronic facial palsy ( $>90$ days after onset).

\begin{tabular}{|c|c|c|c|c|}
\hline & $\begin{array}{c}\text { Acute Palsy } \\
\quad n=697\end{array}$ & $\begin{array}{c}\text { Chronic Palsy } \\
n=523\end{array}$ & & \\
\hline $\begin{array}{l}\text { Parameter } \\
\text { Gender }\end{array}$ & Absolute (N) & Absolute (N) & $X^{2}, \mathrm{df}$ & $p^{*}$ \\
\hline Female & 365 & 348 & $24.7,1$ & $<0.0001$ \\
\hline Male & 332 & 175 & & \\
\hline Side & & & $7.7,2$ & 0.021 \\
\hline Right & 343 & 224 & & \\
\hline Left & 352 & 293 & & \\
\hline Bilateral & 2 & 6 & & \\
\hline Recurrent palsy & & & $4.5,1$ & 0.039 \\
\hline No & 646 & 500 & & \\
\hline Yes & 51 & 23 & & \\
\hline Localization & & & $1.4,2$ & 0.490 \\
\hline peripheral & 692 & 519 & & \\
\hline central & 5 & 3 & & \\
\hline nuclear & 0 & 1 & & \\
\hline Etiology & & & $69.3,6$ & $<0.0001$ \\
\hline idiopathic & 306 & 207 & & \\
\hline Iatrogenic $* *$ & 197 & 139 & & \\
\hline infectious/inflammatory & 104 & 94 & & \\
\hline traumatic & 19 & 24 & & \\
\hline neoplastic, benign & 11 & 31 & & \\
\hline neoplastic, malignant & 0 & 3 & & \\
\hline \multirow[t]{2}{*}{ congenital } & 0 & 25 & & \\
\hline & Mean \pm SD & Mean \pm SD & $\mathrm{T}, \mathrm{df}$ & $p$ \\
\hline $\begin{array}{c}\text { Age (years) at initial } \\
\text { diagnosis }\end{array}$ & $53.0 \pm 18.6$ & $41.2 \pm 20.3$ & $10.6,1218$ & $<0.0001$ \\
\hline $\begin{array}{l}\text { Age (years) at initial } \\
\text { presentation }\end{array}$ & $53.0 \pm 18.6$ & $46.4 \pm 18.2$ & $6.2,1218$ & $<0.0001$ \\
\hline $\begin{array}{l}\text { Interval (years) onset to } \\
\text { initial presentation }\end{array}$ & $0.02 \pm 0.04$ & $5.3 \pm 9.2$ & $-15.3,1218$ & $<0.0001$ \\
\hline
\end{tabular}

* Significant $p$-values $(p<0.05)$ in bold for nominal data due to Pearson's chi-square test for two subgroups or due to Fisher's exact test for more than two subgroups and for metric data due to $t$-test; ${ }^{* *}$ post-operative or post-radiotherapy; $\mathrm{SD}=$ standard.

\subsection{Diagnostics}

The need for diagnostic tests was different in the acute and chronic palsy groups for all analyzed diagnostics (nearly all $p<0.001$; Table 2; data for all patients together are in Table S2). Imaging (neck sonography, magnetic resonance imaging, computed tomography) and serology were relevant for acute but not chronic cases. In contrast, since its introduction, facial sonography has been important for decision-making in chronic flaccid cases. Classical topography tests were predominately needed in acute cases. Standardized facial photo series were very important in all cases but even more important in chronic cases to document the changes during follow-up and to allow controlled facial grading. During follow-up, electrophysiological tests were by far the most frequently repeated investigation (Table S2). The mean number of visits was $2.6 \pm 3.6$ (range: $1-52$ ). If the patients were referred in the acute phase, the mean treatment time in the center was $0.9 \pm 1.9$ years. The treatment time for patients with chronic palsy was $1.4 \pm 1.9$ years. 
Table 2. Comparison of necessary diagnostics in the case of acute facial palsy ( $\leq 90$ days after onset) versus chronic facial palsy ( $>90$ days after onset).

\begin{tabular}{|c|c|c|c|c|}
\hline & $\begin{array}{c}\text { Acute Palsy } \\
n=697\end{array}$ & $\begin{array}{c}\text { Chronic Palsy } \\
n=523\end{array}$ & & \\
\hline Parameter & Absolute (N) & Absolute (N) & $X^{2}, \mathrm{df}$ & $p^{*}$ \\
\hline Sonography of the neck & & & $364.2,1$ & $<0.0001$ \\
\hline Yes & 451 & 54 & & \\
\hline No & 246 & 469 & & \\
\hline $\begin{array}{l}\text { Sonography of the facial } \\
\text { muscles }\end{array}$ & & & $51.5,1$ & $<0.0001$ \\
\hline Yes & 65 & 128 & & \\
\hline No & 632 & 395 & & \\
\hline $\begin{array}{l}\text { Magnet resonance } \\
\text { imaging, cranial }\end{array}$ & & & $38.2,1$ & $<0.0001$ \\
\hline Yes & 112 & 25 & & \\
\hline No & 585 & 498 & & \\
\hline $\begin{array}{l}\text { Computed tomography, } \\
\text { cranial }\end{array}$ & & & $44.2,1$ & $<0.0001$ \\
\hline Yes & 80 & 8 & & \\
\hline No & 617 & 515 & & \\
\hline Facial electrophysiology & & & $5.1,1$ & 0.024 \\
\hline Yes & 596 & 470 & & \\
\hline No & 101 & 53 & & \\
\hline Facial photo series & & & $31.6,1$ & $<0.0001$ \\
\hline Yes & 613 & 503 & & \\
\hline No & 79 & 14 & & \\
\hline Audiometry & & & $10.9,1$ & 0.001 \\
\hline Yes & 508 & 335 & & \\
\hline No & 189 & 188 & & \\
\hline Tympanometry & & & $18.3,1$ & $<0.0001$ \\
\hline Yes & 496 & 311 & & \\
\hline No & 201 & 212 & & \\
\hline Stapedius reflex test & & & $14.3,1$ & $<0.0001$ \\
\hline Yes & 482 & 307 & & \\
\hline No & 215 & 216 & & \\
\hline Gustatory test & & & $47.3,1$ & $<0.0001$ \\
\hline Yes & 477 & 256 & & \\
\hline No & 220 & 267 & & \\
\hline Vestibular tests & & & $101.9,1$ & $<0.0001$ \\
\hline Yes & 484 & 212 & & \\
\hline No & 213 & 311 & & \\
\hline Schirmer test & & & $47.1,1$ & $<0.0001$ \\
\hline Yes & 488 & 233 & & \\
\hline No & 249 & 290 & & \\
\hline Serology & & & $454.5,1$ & $<0.0001$ \\
\hline Yes & 429 & 12 & & \\
\hline No & 268 & 511 & & \\
\hline
\end{tabular}

* Significant $p$-values $(p<0.05)$ in bold; Pearson's chi-square test.

\subsection{Treatment}

Drug treatment was the most important type of therapy for patients with acute facial palsy (Table 3). Glucocorticoid, acyclovir, the combination of glucocorticoid \pm acyclovir, and antibiotics, if indicated, were started with a median time of 1-2 days after onset (Table S3). Conservative eye care (eye moisture chamber, eye drops/ointment) as well as instructions for facial exercises and facial care at home were predominately used for patients with acute palsy. Upper eyelid weight surgery was important for patients with severe acute and chronic flaccid palsy. Other eyelid surgery was predominately reserved for chronic cases. Facial-facial nerve suture and facial nerve interpositional graft were important for acute cases with severe degenerative lesions without the possibility for spontaneous regeneration. Hypoglossal-facial-nerve jump suture was the predominant choice out of the nerve surgery techniques for chronic flaccid cases. Some patients had received surgery before referral to the center ( $n=71$, eyelid surgery; $n=26$, facial nerve surgery; $n=11$, sling surgery). Three patients were referred to other facial specialists $(n=1$; Masseteric-facial nerve suture plus facial cross-face suture; $n=1$; facial cross-face suture; 
$n=1$, temporalis muscle mini-transfer). Electrotherapy, botulinum toxin injection, and facial EMG biofeedback training were important options in the chronic phase of the disease, the former procedure for chronic flaccid cases and the latter two procedures for patients with facial synkinesis.

Table 3. Comparison of treatment in the case of acute facial palsy ( $\leq 90$ days after onset) versus chronic facial palsy ( $>90$ days after onset).

\begin{tabular}{|c|c|c|c|c|}
\hline & $\begin{array}{l}\text { Acute Palsy } \\
\quad n=697\end{array}$ & $\begin{array}{c}\text { Chronic Palsy } \\
\quad n=523\end{array}$ & & \\
\hline Parameter & Absolute (N) & Absolute (N) & $X^{2}, \mathrm{df}$ & $p^{*}$ \\
\hline Glucocorticoids & & & $760.0,1$ & $<0.0001$ \\
\hline Yes & 564 & 7 & & \\
\hline No & 133 & 516 & & \\
\hline Acyclovir & & & $413.5,1$ & $<0.0001$ \\
\hline Yes & 384 & 2 & & \\
\hline No & 313 & 521 & & \\
\hline Antibiotics & & & $39.9,1$ & $<0.0001$ \\
\hline Yes & 60 & 3 & & \\
\hline No & 637 & 520 & & \\
\hline \multicolumn{5}{|l|}{ Facial nerve reconstruction } \\
\hline $\begin{array}{l}\text { Facial nerve reconstruction, } \\
\text { any }\end{array}$ & 16 & 33 & $14.7,1$ & 0.001 \\
\hline Facial-facial nerve suture & 2 & 2 & $0.01,1$ & 1.000 \\
\hline $\begin{array}{l}\text { Facial nerve interpositional } \\
\text { graft }\end{array}$ & 6 & 2 & $1.1,1$ & 0.478 \\
\hline $\begin{array}{l}\text { Hypoglossal-facial-nerve } \\
\text { jump suture }\end{array}$ & 10 & 31 & $18.6,1$ & $<0.0001$ \\
\hline \multicolumn{5}{|l|}{ Muscle and sling plasty } \\
\hline Temporal muscle transfer & 0 & 1 & $1.3,1$ & 0.429 \\
\hline $\begin{array}{l}\text { Sling plasty angle of the } \\
\text { mouth }\end{array}$ & 4 & 12 & $6-8,1$ & 0.011 \\
\hline \multicolumn{5}{|l|}{ Eyelid surgery } \\
\hline Upper eyelid weight & 31 & 46 & $9.6,1$ & 0.003 \\
\hline Tarsorrhaphy & 1 & 9 & $13.2,1$ & 0.001 \\
\hline Kanthopexy & 3 & 9 & $8.4,1$ & 0.006 \\
\hline Brow plasty & 7 & 15 & $10.8,1$ & 0.002 \\
\hline Blepharoplasty & 2 & 13 & $17.5,1$ & 0.001 \\
\hline Lower lid plasty & 8 & 17 & $12.1,1$ & 0.013 \\
\hline \multicolumn{5}{|l|}{$\begin{array}{c}\text { Non-surgical adjuvant } \\
\text { therapy }\end{array}$} \\
\hline $\begin{array}{l}\text { Physical therapy/speech } \\
\text { therapy }\end{array}$ & 55 & 47 & $0.5,1$ & 0.531 \\
\hline Electrotherapy & 11 & 47 & $51.5,1$ & $<0.0001$ \\
\hline Botulinumtoxin injection & 41 & 132 & $92.0,1$ & $<0.0001$ \\
\hline Eye moisture chamber & 423 & 12 & $444.1,1$ & $<0.0001$ \\
\hline Eye drops/ointment & 430 & 20 & $430.0,1$ & $<0.0001$ \\
\hline Facial exercises at home & 441 & 14 & $469.1,1$ & $<0.0001$ \\
\hline $\begin{array}{c}\text { Facial EMG biofeedback } \\
\text { training }\end{array}$ & 31 & 255 & $326.9,1$ & $<0.0001$ \\
\hline
\end{tabular}

* Significant $p$-values $(p<0.05)$ in bold; Pearson's chi-square test.

\subsection{Facial Nerve Function at Initial and Last Visits}

Facial nerve function in patients with acute or chronic facial palsy was highly variable (Table S4). The relation between incomplete and complete facial palsy in the acute phase was 5:1. Post-paralytic synkinesis was the dominant status of patients referred in the chronic phase. The number of visits to the center varied from 1 to 52 . The average interval between initial and last presentation was $1.1 \pm 1.9$ years. At the initial visit, male patients had significantly worse facial function than female patients $(p<0.0001)$, but in all PROMs, they had better facial-specific (FDI, FaCE) and general (SF-36) quality of life $(p<0.0001$ for nearly all domains; Table 4 ). Patients $>50$ years of age had a worse facial function and lower PROM values in most subdomains ( $p<0.0001$ for nearly all domains). Patients with chronic facial palsy (flaccid and synkinesis) had better facial function than patients with acute palsy but lower FDI, FaCE, and SF-36 values in most subdomains. Patients with facial palsy related to a tumor had the lowest quality of life values. At the last visit, the gender influence was smaller; female patients displayed a lower quality of life in fewer subdomains (Table 5). Patients $>50$ years of age and patients with chronic palsy still showed a lower quality of life in most subdomains. 
Table 4. Initial facial function of patients by demographics and etiology.

\begin{tabular}{|c|c|c|c|c|c|c|c|}
\hline Parameter & $\begin{array}{c}\text { SI, Total } \\
(\text { Mean } \pm \text { SD) }\end{array}$ & $\begin{array}{l}\text { FDI, Physical } \\
\text { (Mean } \pm \text { SD) }\end{array}$ & $\begin{array}{c}\text { FDI, Social } \\
(\text { Mean } \pm \text { SD) }\end{array}$ & $\begin{array}{c}\text { FDI, Total } \\
(\text { Mean } \pm \text { SD) }\end{array}$ & $\begin{array}{c}\text { FaCE, Total } \\
(\text { Mean } \pm \text { SD) }\end{array}$ & $\begin{array}{c}\text { SF-36, PFI } \\
(\text { Mean } \pm \text { SD) }\end{array}$ & $\begin{array}{r}\text { SF-36, GHP } \\
(\text { Mean } \pm \text { SD) }\end{array}$ \\
\hline \multicolumn{8}{|l|}{ Gender } \\
\hline Female & $4.5 \pm 3.0$ & $64.2 \pm 20.4$ & $66.2 \pm 21.1$ & $65.1 \pm 18.0$ & $57.3 \pm 22.8$ & $80.3 \pm 26.4$ & $57.5 \pm 21.0$ \\
\hline Male & $5.1 \pm 3.0$ & $69.7 \pm 20.1$ & $74.5 \pm 20.4$ & $72.0 \pm 17.2$ & $67.7 \pm 21.7$ & $85.0 \pm 21.6$ & $62.8 \pm 20.8$ \\
\hline $\mathrm{T}, \mathrm{df}$ & $-3.6,1218$ & $-4.0,846$ & $-5.9,841$ & $-5.9,847$ & $-6.4,829$ & $-2.8,811$ & $-3.5,796$ \\
\hline$p^{*}$ & $<0.0001$ & $<0.0001$ & $<0.0001$ & $<0.0001$ & $<0.0001$ & 0.005 & $<0.0001$ \\
\hline \multicolumn{8}{|l|}{ Age at first presentation } \\
\hline$<$ median 50 years & $4.4 \pm 2.9$ & $69.6 \pm 20.0$ & $70.5 \pm 20.6$ & $70.0 \pm 17.9$ & $62.7 \pm 22.1$ & $88.9 \pm 20.3$ & $61.4 \pm 21.4$ \\
\hline$\geq$ median 50 years & $5.0 \pm 3.1$ & $63.1 \pm 20.4$ & $68.5 \pm 20.9$ & $65.5 \pm 17.9$ & $60.8 \pm 23.9$ & $72.6 \pm 25.4$ & $58.2 \pm 19.9$ \\
\hline $\mathrm{T}, \mathrm{df}$ & $-3.6,1218$ & $4.7,846$ & $1.8,841$ & $3.7,847$ & $1.6,829$ & $8.5,811$ & $4.4,796$ \\
\hline$p^{*}$ & $<0.0001$ & $<0.0001$ & 0.065 & $<0.0001$ & 0.118 & $<0.0001$ & $<0.0001$ \\
\hline \multicolumn{8}{|l|}{$\begin{array}{l}\text { Interval onset to first } \\
\text { presentation }\end{array}$} \\
\hline $\begin{array}{c}\leq 90 \text { days (acute facial } \\
\text { palsy) }\end{array}$ & $5.1 \pm 2.9$ & $69.6 \pm 21.1$ & $75.9 \pm 18.1$ & $72.6 \pm 17.3$ & $72.3 \pm 23.2$ & $80.2 \pm 24.3$ & $59.8 \pm 20.1$ \\
\hline $\begin{array}{l}>90 \text { days (chronic facial } \\
\text { palsy) }\end{array}$ & $4.2 \pm 3.1$ & $63.7 \pm 19.5$ & $64.2 \pm 21.2$ & $63.9 \pm 17.6$ & $53.8 \pm 19.2$ & $80.8 \pm 24.7$ & $58.8 \pm 22.2$ \\
\hline $\mathrm{T}, \mathrm{df}$ & $5.3,1218$ & $4.0,846$ & $8.0,841$ & $7.0,847$ & $12.5,829$ & $-1.1,811$ & $2.3,796$ \\
\hline$p^{*}$ & $<0.0001$ & $<0.0001$ & $<0.0001$ & $<0.0001$ & $<0.0001$ & 0.253 & 0.023 \\
\hline \multicolumn{8}{|l|}{ Etiology } \\
\hline idiopathic & $4.7 \pm 2.8$ & $68.7 \pm 20.8$ & $70.4 \pm 21.3$ & $69.3 \pm 18.4$ & $65.5 \pm 22.8$ & $83.2 \pm 22.7$ & $59.8 \pm 20.9$ \\
\hline iatrogenic & $4.4 \pm 3.1$ & $64.6 \pm 18.3$ & $70.2 \pm 19.9$ & $67.4 \pm 16.5$ & $59.4 \pm 21.5$ & $78.4 \pm 24.5$ & $61.7 \pm 20.2$ \\
\hline infectious/inflammatory & $4.8 \pm 2.9$ & $60.3 \pm 21.4$ & $64.7 \pm 20.2$ & $62.6 \pm 18.8$ & $57.3 \pm 25.2$ & $74.4 \pm 28.1$ & $55.3 \pm 23.7$ \\
\hline traumatic & $4.6 \pm 3.2$ & $72.1 \pm 21.8$ & $70.0 \pm 20.9$ & $70.8 \pm 17.5$ & $60.8 \pm 22.4$ & $76.4 \pm 31.6$ & $54.9 \pm 18.5$ \\
\hline neoplastic, benign & $7.9 \pm 2.4$ & $62.7 \pm 14.7$ & $64.8 \pm 16.7$ & $63.8 \pm 12.4$ & $47.2 \pm 12.5$ & $83.5 \pm 17.4$ & $56.5 \pm 17.2$ \\
\hline neoplastic, malignant & $8.3 \pm 1.2$ & $60.0 \pm 0$ & $82.0 \pm 8.5$ & $71.0 \pm 4.2$ & $45.8 \pm 20.0$ & $90.0 \pm 14.4$ & $61.0 \pm 1.4$ \\
\hline congenital & $4.9 \pm 2.7$ & $87.5 \pm 13.2$ & $86.8 \pm 13.7$ & $87.1 \pm 11.2$ & $72.8 \pm 16.2$ & $100.0 \pm 0$ & $75.8 \pm 24.6$ \\
\hline $\mathrm{F}, \mathrm{df}$ & $9.5,6$ & $7.0,6$ & $3.4,6$ & $6.6,6$ & $6.5,6$ & $4.2,6$ & $3.8,6$ \\
\hline$p^{*}$ & $<0.0001$ & 0.003 & 0.001 & $<0.0001$ & $<0.0001$ & $<0.0001$ & 0.001 \\
\hline
\end{tabular}

$\mathrm{SD}$ = standard deviation; SI = Stennert index; FDI = Facial Disability index; FaCE = Facial Clinimetric Evaluation Scale; SF-36 = 36-Item Short Form Survey; PF = physical function subdomain; GH = general health subdomain * Significant $p$-values $(p<0.05)$ in bold; $t$-test for comparison of two subgroups; univariate ANOVA for more than two subgroups.

Table 5. Last facial function of patients by demographics and etiology.

\begin{tabular}{|c|c|c|c|c|c|c|c|}
\hline Parameter & $\begin{array}{c}\text { SI, Total } \\
(\text { Mean } \pm \text { SD) }\end{array}$ & $\begin{array}{l}\text { FDI, Physical } \\
\text { (Mean } \pm \text { SD) }\end{array}$ & $\begin{array}{c}\text { FDI, Social } \\
(\text { Mean } \pm \text { SD) }\end{array}$ & $\begin{array}{c}\text { FDI, Total } \\
\text { (Mean } \pm \text { SD) }\end{array}$ & $\begin{array}{c}\text { FaCE, Total } \\
(\text { Mean } \pm \text { SD) }\end{array}$ & $\begin{array}{c}\text { SF-36, PFI } \\
(\text { Mean } \pm \text { SD) }\end{array}$ & $\begin{array}{c}\text { SF-36, GHP } \\
\text { (Mean } \pm \text { SD) }\end{array}$ \\
\hline \multicolumn{8}{|l|}{ Gender } \\
\hline Female & $2.0 \pm 2.5$ & $74.7 \pm 17.4$ & $74.7 \pm 17.0$ & $74.7 \pm 15.2$ & $64.7 \pm 20.1$ & $83.4 \pm 22.6$ & $63.3 \pm 21.2$ \\
\hline Male & $2.2 \pm 2.9$ & $78.0 \pm 17.6$ & $79.0 \pm 17.8$ & $84.4 \pm 26.5$ & $73.2 \pm 19.7$ & $82.1 \pm 25.7$ & $61.6 \pm 20.4$ \\
\hline $\mathrm{T}, \mathrm{df}$ & $-0.6,1072$ & $-1.6,621$ & $-2.6,617$ & $-2.5,617$ & $-4.0,618$ & $-0.2,618$ & $-0.1,618$ \\
\hline$p^{*}$ & 0.519 & 0.105 & 0.011 & 0.014 & $<0.0001$ & 0.781 & 0.679 \\
\hline \multicolumn{8}{|l|}{ Age at first presentation } \\
\hline$<$ median 50 years & $2.3 \pm 2.7$ & $79.3 \pm 16.3$ & $76.7 \pm 17.2$ & $78.1 \pm 15.1$ & $69.1 \pm 19.4$ & $90.8 \pm 17.6$ & $65.5 \pm 21.2$ \\
\hline$>$ median 50 years & $1.9 \pm 2.7$ & $71.8 \pm 18.0$ & $75.5 \pm 17.6$ & $73.7 \pm 56.8$ & $65.9 \pm 21.2$ & $74.9 \pm 26.4$ & $59.6 \pm 20.2$ \\
\hline $\mathrm{T}, \mathrm{df}$ & $2.5,1072$ & $4.4,621$ & $0.6,617$ & $2.6,617$ & $1.1,618$ & $6.4,618$ & $3.7,618$ \\
\hline$p^{*}$ & 0.012 & $<0.0001$ & 0.499 & 0.009 & 0.295 & $<0.0001$ & $<0.0001$ \\
\hline \multicolumn{8}{|l|}{$\begin{array}{l}\text { Interval onset to first } \\
\text { presentation }\end{array}$} \\
\hline $\begin{array}{c}\leq 90 \text { days (acute facial } \\
\text { palsy) }\end{array}$ & $1.2 \pm 2.3$ & $80.1 \pm 18.1$ & $80.1 \pm 16.1$ & $85.4 \pm 23.1$ & $77.6 \pm 21.1$ & $84.0 \pm 22.6$ & $64.2 \pm 25.7$ \\
\hline $\begin{array}{l}>90 \text { days (chronic facial } \\
\text { palsy) }\end{array}$ & $3.6 \pm 2.7$ & $73.3 \pm 16.7$ & $73.8 \pm 17.7$ & $73.6 \pm 15.2$ & $62.7 \pm 17.9$ & $81.1 \pm 25.7$ & $60.2 \pm 19.5$ \\
\hline $\mathrm{T}, \mathrm{df}$ & $-15.2,1072$ & $3.8,621$ & $2.4,617$ & $3.6,617$ & $8.2,618$ & $-0.7,618$ & $0.2,618$ \\
\hline$p^{*}$ & $<0.0001$ & $<0.0001$ & 0.017 & $<0.0001$ & $<0.0001$ & 0.533 & 0.812 \\
\hline \multicolumn{8}{|l|}{ Etiology } \\
\hline idiopathic & $1.4 \pm 2.0$ & $77.6 \pm 17.4$ & $77.9 \pm 16.4$ & $77.8 \pm 14.7$ & $72.9 \pm 18.6$ & $86.3 \pm 21.6$ & $66.7 \pm 22.4$ \\
\hline iatrogenic & $2.5 \pm 2.9$ & $75.1 \pm 17.4$ & $76.2 \pm 16.9$ & $82.4 \pm 17.3$ & $62.7 \pm 20.9$ & $83.2 \pm 19.9$ & $58.2 \pm 14.9$ \\
\hline infectious/inflammatory & $2.0 \pm 2.3$ & $74.0 \pm 16.8$ & $73.7 \pm 19.7$ & $77.9 \pm 14.4$ & $65.9 \pm 20.1$ & $70.5 \pm 14.0$ & $54.8 \pm 24.6$ \\
\hline traumatic & $3.0 \pm 3.2$ & $82.2 \pm 18.1$ & $77.5 \pm 13.5$ & $79.8 \pm 13.6$ & $73.3 \pm 17.9$ & $92.0 \pm 17.9$ & $77.0 \pm 15.4$ \\
\hline neoplastic, benign & $7.4 \pm 2.0$ & $60.9 \pm 14.8$ & $65.2 \pm 18.3$ & $63.0 \pm 14.3$ & $49.5 \pm 17.6$ & $84.2 \pm 22.2$ & $65.8 \pm 15.0$ \\
\hline neoplastic, malignant & $10 \pm 0$ & $75.0 \pm 0$ & $80.0 \pm 0$ & $77.5 \pm 0$ & $48.3 \pm 0$ & NA & NA \\
\hline congenital & $4.2 \pm 2.8$ & $96.3 \pm 2.5$ & $92.8 \pm 3.6$ & $94.5 \pm 2.0$ & $83.3 \pm 4.1$ & $95.0 \pm 0$ & $62.0 \pm 0$ \\
\hline $\mathrm{F}, \mathrm{df}$ & $42.4,6$ & $3.3,6$ & $1.7,6$ & $3.0,6$ & $5.1,6$ & $2.0,6$ & $2.8,6$ \\
\hline$p^{*}$ & $<0.0001$ & 0.003 & 0.108 & 0.008 & $<0.0001$ & 0.066 & 0.010 \\
\hline
\end{tabular}

$\mathrm{SD}=$ standard deviation; $\mathrm{SI}=$ Stennert index $; \mathrm{FDI}=$ Facial Disability index $; \mathrm{FaCE}=$ Facial Clinimetric Evaluation Scale; SF-36 = 36-Item Short Form Survey; PFI = physical function subdomain; GHP = general health subdomain; * significant $p$-values $(p<0.05)$ in bold; $t$-test for comparison of two subgroups; univariate ANOVA for more than two subgroups.

\subsection{Outcome}

The interval between first and last presentation for all patients was $1.1 \pm 1.9$ years (acute palsy: $0.9 \pm 1.9$; chronic palsy: $1.4 \pm 1.9$ years). The outcome measures for the 
first and last visit for the different treatments are shown for the Stennert index and the FDI in Table 6 and for the FaCE and the SF-36 in Table 7. The absolute improvement of facial grading for selected subgroups is presented in Figure 1. Overall, facial grading improved highly significantly $(p<0.001)$ for patients with acute or chronic palsy during the treatment in the center. The changes of the PROMs are presented in Figure 2. Treatment with glucocorticoids, acyclovir, and antibiotics was followed by a highly significant improvement of all outcome measures $(p<0.001)$. The effect was less notable for the SF-36 $(p<0.05)$. In case of chronic flaccid palsy, hypoglossal-facial-nerve jump suture, any eyelid surgery, and especially an upper eyelid weight led to significant improvement of facial motor function $(p<0.001)$ and facial-specific quality of life (FDI, FaCE; $p<0.05)$, but not to an improvement in the general quality of life (SF-36; $p>0.05)$. Conservative measures, such as physical therapy, botulinum toxin injections, special facial exercises at home, or facial EMG feedback training, also led to highly significant improvements according to facial grading, FDI, and FaCE (mostly, $p<0.001$ ). Again, the effect was not highly significant for the SF-36. There was one exception, general quality of life was significantly improved when using botulinum toxin injections for facial synkinesis $(p<0.001)$. In addition, Table S5 regards the improvement from the perspective of acute and chronic facial palsy separately and for the PROM subdomains. Treatment of patients with acute palsy improved in nearly all subdomains highly significantly $(p<0.001)$, for general quality of life significantly (SF-36; $p<0.05)$. Patients with chronic facial palsy showed highly significant improvements (mostly $p<0.001)$, except for some SF-36 subdomains $(p>0.05)$. Glucocorticoids alone for acute facial palsy were effective in regard to the improvement of facial grading, FDI, and FaCE, but not for the SF-36 subdomains (Table S6). Botulinum toxin injections for facial synkinesis were also effective, seen in the improvement of facial grading and of FDI and in some subdomains of FaCE and SF-36 (mostly $p<0.001$ ). The improvement for patients with facial synkinesis was even greater after EMG facial biofeedback training (mostly $p<$ 0.001; Table S7). The improvement was less pronounced for surgical procedures, analyzed for the larger subgroups of hypoglossal-facial jump surgery and eyelid surgery: Facial grading was significantly improved, along with some FDI subdomains and a few FaCE subdomains, and only the SF-36 physical functioning subdomain after hypoglossal-facial jump surgery.

Finally, the recovery rates after acute facial palsy were analyzed. Univariate analysis showed that idiopathic etiology, incomplete palsy, low initial Stennert grading, high initial FDI or FaCE, no pathological spontaneous activity in EMG, normal stapedial reflex, prednisolone, or combined prednisolone and acyclovir treatment were beneficial parameters associated to higher complete recovery rates (Figure S3, Table S8; all $p<0.05$ ). Multivariate modeling (Table S9) showed that initial better facial grading (hazard ratio (HR) 1.571; confidence interval (CI) 1.026 to 2.404, $p=0.038$ ) and FaCE total score (HR 2.653; CI 1.519 to $4.635 ; p=0.001$ ) predicted higher probability of complete recovery. Idiopathic etiology was related to better outcome (HR 1.320; CI 1.000 to $1.742, p=0.050$ ) and an iatrogenic lesion to worse outcome (HR 0.485; CI 0.354 to $0.665 ; p<0.0001$ ). Out of the diagnostic tests, a normal stapedial reflex remained an independent predictor of better outcome (HR 2.077; CI 1.498 to 2.88; $p<0.0001)$. Concerning acute therapy, prednisolone alone or in combination was related to better outcome (HR 3.614; CI 2.124 to $6.149 ; p<0.0001$ ). 
Table 6. Comparison of outcome measures using the Stennert index (sum) and the Facial Disability index (total) between first and last visits *

\begin{tabular}{|c|c|c|c|c|c|c|c|c|}
\hline & \multicolumn{5}{|c|}{ Stennert Index, Sum } & \multicolumn{3}{|c|}{ Facial Disability Index, Total } \\
\hline & First Visit Mean \pm SD & Last Visit Mean \pm SD & $\mathrm{T}, \mathrm{df}$ & $p$ & First Visit Mean \pm SD & Last Visit Mean \pm SD & $\mathrm{T}, \mathrm{df}$ & $p^{* *}$ \\
\hline All & $4.8 \pm 3.0$ & $2.1 \pm 2.7$ & $28.8,1073$ & $<0.0001$ & $64.8 \pm 16.6$ & $76.0 \pm 16.7$ & $-14.7,604$ & $<0.0001$ \\
\hline Glucocorticoids & $5.1 \pm 2.7$ & $1.1 \pm 2.1$ & $32.1,570$ & $<0.0001$ & $68.3 \pm 6.9$ & $87.4 \pm 70.1$ & $-82,327$ & 0.006 \\
\hline Acyclovir & $5.6 \pm 2.5$ & $1.0 \pm 2.0$ & $32.1,384$ & $<0.0001$ & $68.7 \pm 16.4$ & $81.0 \pm 15.0$ & $-5.2,170$ & $<0.001$ \\
\hline $\begin{array}{l}\text { Glucocorticoids } \pm \\
\text { Acyclovir }\end{array}$ & $5.7 \pm 2.5$ & $1.0 \pm 2.0$ & $32.8,380$ & $<0.0001$ & $62.5 \pm 19.4$ & $80.5 \pm 17.6$ & $-6.0,180$ & $<0.0001$ \\
\hline Antibiotics & $5.6 \pm 2.7$ & $1.8 \pm 2.9$ & $10.0,62$ & $<0.0001$ & $65.9 \pm 12.6$ & $78.9 \pm 27.4$ & $-3.8,18$ & 0.021 \\
\hline $\begin{array}{l}\text { Facial nerve } \\
\text { reconstruction, any }\end{array}$ & $8.6 \pm 1.7$ & $6.8 \pm 2.6$ & $5.1,48$ & $<0.0001$ & $67.3 \pm 16.7$ & $71.3 \pm 19.9$ & $-2.4,32$ & 0.019 \\
\hline $\begin{array}{l}\text { Facial nerve } \\
\text { interpositional graft }\end{array}$ & $8.3 \pm 1.5$ & $5.9 \pm 3.0$ & $2.1,7$ & 0.074 & $73.5 \pm 7.4$ & $73.5 \pm 8.7$ & 0,3 & 1.000 \\
\hline $\begin{array}{l}\text { Hypoglossal-facial- } \\
\text { nerve jump } \\
\text { suture }\end{array}$ & $8.7 \pm 1.3$ & $6.8 \pm 2.5$ & $4.9,40$ & $<0.0001$ & $66.2 \pm 16.2$ & $77.5 \pm 20.0$ & $-2.4,28$ & 0.020 \\
\hline $\begin{array}{l}\text { Sling plasty angle of } \\
\text { the mouth }\end{array}$ & $8.2 \pm 2.2$ & $9.3 \pm 1.3$ & $-2.5,15$ & 0.023 & $62.8 \pm 14.3$ & $52.4 \pm 10.7$ & $21.2,13$ & 0.056 \\
\hline Eyelid surgery, any & $7.2 \pm 2.9$ & $6.1 \pm 3.1$ & $3.7,91$ & $<0.0001$ & $61.6 \pm 17.1$ & $64.0 \pm 19.4$ & $-2.1,52$ & 0.042 \\
\hline Upper eyelid weight & $7.7 \pm 2.5$ & $6.4 \pm 3.0$ & $4.1,76$ & $<0.0001$ & $61.2 \pm 17.9$ & $63.2 \pm 20.6$ & $-1.8,42$ & 0.072 \\
\hline Tarsorrhaphy & $7.0 \pm 2.5$ & $5.9 \pm 2.8$ & $0.9,8$ & 0.384 & $59.0 \pm 14.7$ & $62.1 \pm 16.3$ & $-0.6,7$ & 0.550 \\
\hline Brow plasty & $7.2 \pm 3.1$ & $6.7 \pm 3.6$ & $0.8,21$ & 0.451 & $67.1 \pm 15.4$ & $60.5 \pm 23.8$ & $0.8,12$ & 0.427 \\
\hline Blepharoplasty & $4.4 \pm 3.6$ & $4.5 \pm 2.8$ & $-0.1,14$ & 0.896 & $57.3 \pm 17.3$ & $65.3 \pm 16.6$ & $-2.6,12$ & 0.025 \\
\hline $\begin{array}{l}\text { Lower lid plasty } \\
\text { Physical }\end{array}$ & $8.6 \pm 1.9$ & $7.8 \pm 2.3$ & $1.7,24$ & 0.100 & $59.5 \pm 23.2$ & $57.7 \pm 25.2$ & $-1.4,12$ & 0.811 \\
\hline $\begin{array}{c}\text { therapy/speech } \\
\text { therapy }\end{array}$ & $5.9 \pm 3.0$ & $3.3 \pm 3.0$ & $7.9,96$ & $<0.0001$ & $62.5 \pm 14.9$ & $70.3 \pm 19.4$ & $-5.4,65$ & 0.002 \\
\hline Electrotherapy & $7.3 \pm 2.3$ & $5.9 \pm 2.9$ & $4.1,52$ & $<0.0001$ & $62.1 \pm 15.9$ & $70.8 \pm 17.1$ & $3.9,46$ & $<0.0001$ \\
\hline $\begin{array}{l}\text { Botulinumtoxin } \\
\text { injection }\end{array}$ & $3.7 \pm 2.9$ & $2.3 \pm 1.9$ & $6.4,159$ & $<0.0001$ & $60.6 \pm 15.9$ & $72.0 \pm 15.7$ & $-6.7,110$ & $<0.0001$ \\
\hline $\begin{array}{l}\text { Eye moisture } \\
\text { chamber }\end{array}$ & $6.0 \pm 2.4$ & $1.3 \pm 2.4$ & $32.6,434$ & $<0.0001$ & $65.0 \pm 17.8$ & $65.0 \pm 17.8$ & $-2.6,87$ & 0.010 \\
\hline Eye drops/ointment & $5.9 \pm 2.5$ & $1.3 \pm 2.4$ & $32.1,448$ & $<0.0001$ & $65.7 \pm 16.6$ & $78.7 \pm 16.6$ & $-7.7,112$ & $<0.0001$ \\
\hline $\begin{array}{c}\text { Facial exercises at } \\
\text { home }\end{array}$ & $5.4 \pm 2.6$ & $1.0 \pm 2.0$ & $31.8,452$ & $<0.0001$ & $68.7 \pm 16.1$ & $80.8 \pm 16.6$ & $-5.5,85$ & $<0.0001$ \\
\hline $\begin{array}{c}\text { Facial EMG } \\
\text { biofeedback training }\end{array}$ & $3.8 \pm 2.8$ & $2,8 \pm 2.0$ & $7.3,284$ & $<0.0001$ & $63.1 \pm 1.2$ & $74.3 \pm 15.3$ & $-10.2,195$ & $<0.0001$ \\
\hline
\end{tabular}

${ }^{*} n=1073$ patients with at least two visits; ${ }^{* *}$ significant $p$-values $(p<0.05)$ in bold; paired $t$-test; NA = not applicable; SD = standard deviation. 
Table 7. Comparison of outcome measures using FaCE (total score) and SF-36 (general health subdomain) between first and last visits *

\begin{tabular}{|c|c|c|c|c|c|c|c|c|}
\hline & \multicolumn{5}{|c|}{ FaCE, Total Score } & \multicolumn{3}{|c|}{ SF-36, General Health } \\
\hline & First Visit Mean \pm SD & Last Visit Mean \pm SD & $\mathrm{T}, \mathrm{df}$ & $p$ & First Visit Mean \pm SD & Last Visit Mean \pm SD & $\mathrm{T}, \mathrm{df}$ & $p^{* *}$ \\
\hline All & $53.8 \pm 20.0$ & $67.3 \pm 20.8$ & $-15.8,613$ & $<0.0001$ & $58.4 \pm 19.6$ & $61.9 \pm 21.7$ & $-3.7,599$ & $<0.0001$ \\
\hline Glucocorticoids & $60.1 \pm 21.5$ & $80.8 \pm 20.0$ & $-10.2,322$ & $<0.0001$ & $59.0 \pm 16.7$ & $65.0 \pm 21.8$ & $-2.3,309$ & 0.014 \\
\hline Acyclovir & $62.8 \pm 19.4$ & $83.8 \pm 16.9$ & $-8.0,158$ & $<0.0001$ & $58.2 \pm 17.5$ & $65.0 \pm 23.4$ & $-2.3,140$ & 0.028 \\
\hline $\begin{array}{l}\text { Glucocorticoids } \pm \\
\text { Acyclovir }\end{array}$ & $60.5 \pm 20.8$ & $82.6 \pm 17.9$ & $-8.7,177$ & $<0.0001$ & $59.6 \pm 17.5$ & $64.4 \pm 22.9$ & $-2.0,171$ & 0.047 \\
\hline Antibiotics & $53.2 \pm 20.5$ & $80.7 \pm 27.8$ & $-6.9,17$ & $<0.0001$ & $54.4 \pm 24.1$ & $65.4 \pm 19.7$ & $-0.6,14$ & 0.066 \\
\hline $\begin{array}{l}\text { Facial nerve } \\
\text { reconstruction, any }\end{array}$ & $54.9 \pm 19.3$ & $60.6 \pm 18.7$ & $-3.1,32$ & 0.005 & $59.0 \pm 24.3$ & $61.2 \pm 13.5$ & $-0.5,26$ & 0.601 \\
\hline $\begin{array}{l}\text { Facial nerve } \\
\text { interpositional graft }\end{array}$ & $51.7 \pm 11.7$ & $53.0 \pm 8.4$ & $-0.1,3$ & 0.917 & $64.6 \pm 10.4$ & $61.5 \pm 15.6$ & $0.8,3$ & 0.469 \\
\hline $\begin{array}{l}\text { Hypoglossal-facial- } \\
\text { nerve jump } \\
\text { suture }\end{array}$ & $51.0 \pm 18.5$ & $59.3 \pm 18.2$ & $-3.1,28$ & 0.005 & $58.6 \pm 26.9$ & $61.2 \pm 14.9$ & $-0.7,22$ & 0.469 \\
\hline $\begin{array}{l}\text { Sling plasty angle of } \\
\text { the mouth }\end{array}$ & $30.0 \pm 4.7$ & $29.2 \pm 8.3$ & $0.1,2$ & 0.942 & $42.5 \pm 10.6$ & $48.5 \pm 12.0$ & $-0.4,4$ & 0.772 \\
\hline Eyelid surgery, any & $46.9 \pm 18.1$ & $50.7 \pm 21.1$ & $-2.5,51$ & 0.015 & $56.7 \pm 23.9$ & $54.2 \pm 18.3$ & $0.9,47$ & 0.390 \\
\hline Upper eyelid weight & $48.1 \pm 19.8$ & $50.6 \pm 23.1$ & $-2.0,42$ & 0.048 & $54.8 \pm 25.4$ & $53.8 \pm 17.8$ & $0.4,37$ & 0.671 \\
\hline Tarsorrhaphy & $41.7 \pm 15.0$ & $49.7 \pm 14.3$ & $-4.7,6$ & 0.009 & $48.5 \pm 19.1$ & $53.5 \pm 5.0$ & $-0.3,6$ & 0.818 \\
\hline Brow plasty & $53.0 \pm 15.5$ & $55.2 \pm 20.1$ & $-0.4,9$ & 0.675 & $54.7 \pm 10.8$ & $50.7 \pm 18.5$ & $0.9,7$ & 0.475 \\
\hline Blepharoplasty & $44.9 \pm 17.9$ & $49.8 \pm 23.2$ & $-1.1,11$ & 0.300 & $57.4 \pm 14.1$ & $53.0 \pm 19.1$ & $0.8,12$ & 0.462 \\
\hline $\begin{array}{l}\text { Lower lid plasty } \\
\text { Physical }\end{array}$ & $53.0 \pm 21.3$ & $55.0 \pm 25.0$ & $-0.4,12$ & 0.630 & $54.0 \pm 36.2$ & $47.3 \pm 31.6$ & $1.4,9$ & 0.184 \\
\hline $\begin{array}{l}\text { therapy/speech } \\
\text { therapy }\end{array}$ & $52.1 \pm 20.3$ & $62.5 \pm 20.3$ & $-5.9,61$ & $<0.0001$ & $54.7 \pm 14.7$ & $55.8 \pm 12.5$ & $-0.2,56$ & 0.586 \\
\hline Electrotherapy & $45.5 \pm 18.1$ & $54.4 \pm 20.6$ & $-4.7,45$ & $<0.0001$ & $56.0 \pm 18.8$ & $57.1 \pm 21.8$ & $-0.4,44$ & 0.715 \\
\hline $\begin{array}{l}\text { Botulinumtoxin } \\
\text { injection }\end{array}$ & $50.5 \pm 19.7$ & $61.1 \pm 17.1$ & $-6.5,110$ & $<0.0001$ & $53.7 \pm 20.2$ & $62.1 \pm 23.4$ & $-1.9,84$ & $<0.0001$ \\
\hline $\begin{array}{l}\text { Eye moisture } \\
\text { chamber }\end{array}$ & $57.4 \pm 22.2$ & $78.7 \pm 22.2$ & $-9.2,73$ & $<0.0001$ & $58.5 \pm 18.3$ & $66.5 \pm 22.8$ & $-2.6,69$ & 0.013 \\
\hline Eye drops/ointment & $57.2 \pm 21.4$ & $77.6 \pm 22.3$ & $-10.0,108$ & $<0.0001$ & $59.4 \pm 17.4$ & $66.6 \pm 22.9$ & $-1.9,107$ & 0.020 \\
\hline $\begin{array}{c}\text { Facial exercises at } \\
\text { home }\end{array}$ & $63.3 \pm 18.5$ & $81.6 \pm 18.5$ & $-7.4,72$ & $<0.0001$ & $59.5 \pm 16.8$ & $64.7 \pm 22.8$ & $-1.8,60$ & 0.076 \\
\hline $\begin{array}{c}\text { Facial EMG } \\
\text { biofeedback training }\end{array}$ & $52.0 \pm 17.9$ & $63.9 \pm 16.6$ & $-10.7,190$ & $<0.0001$ & $61.2 \pm 21.6$ & $61.5 \pm 19.2$ & $-0.9,127$ & 0.932 \\
\hline
\end{tabular}




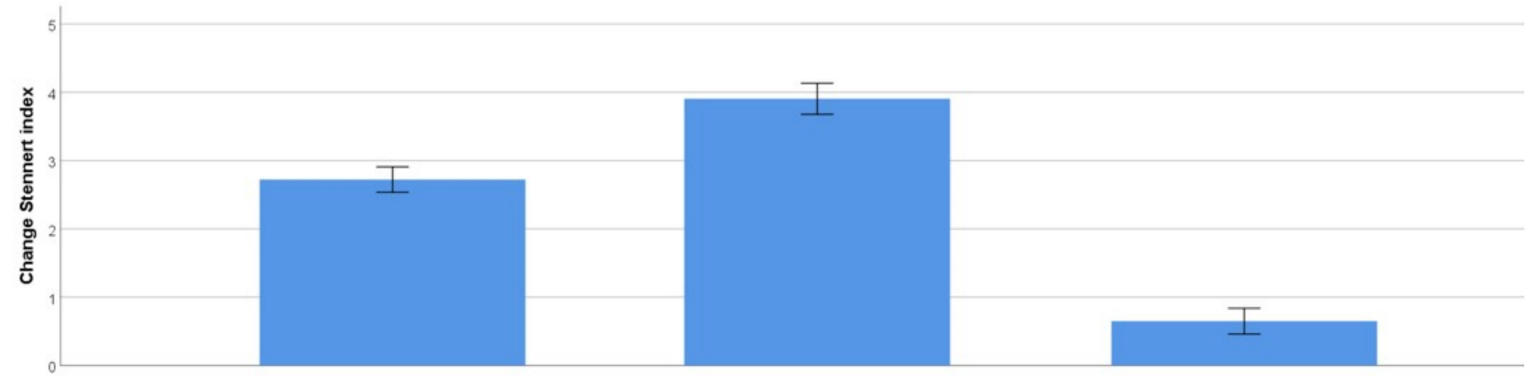

All

Acute palsy

Chronic palsy
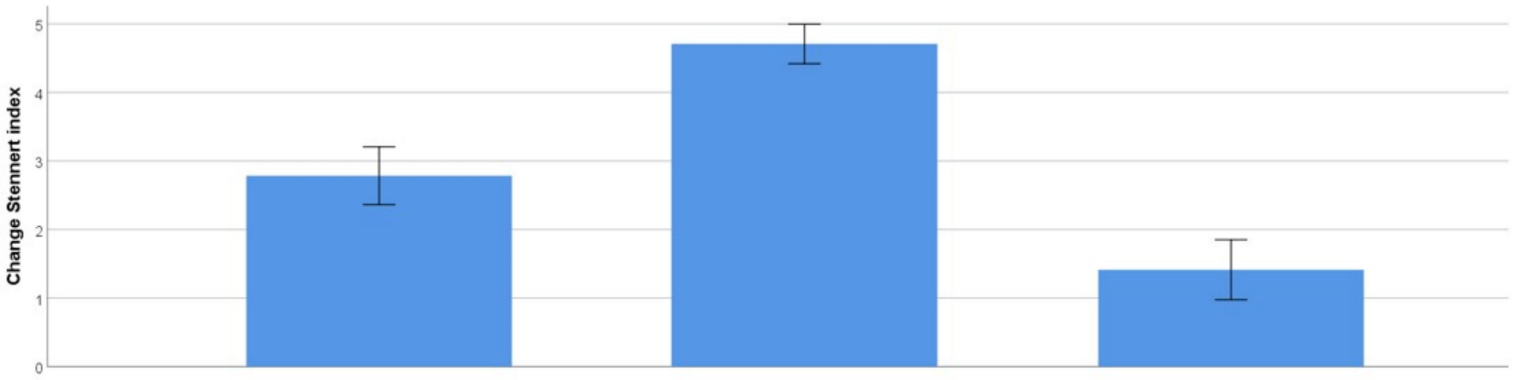

Glucocorticoids alone

Glucocorticoids and acyclovir

Botulinumtoxin

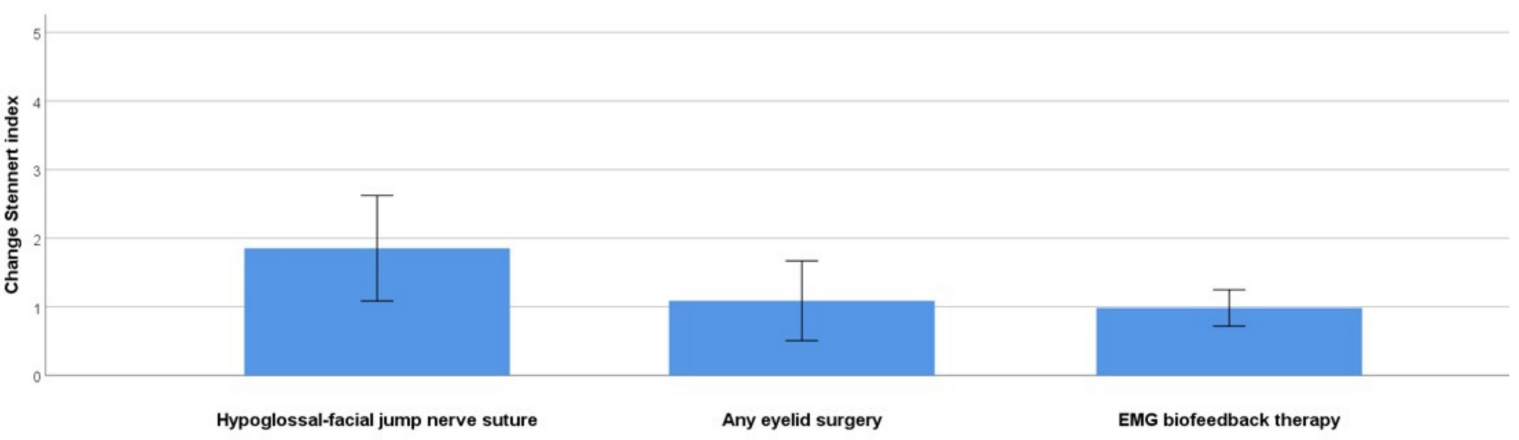

Figure 1. Absolute improvement of facial function between initial and last evaluation in the Facial Nerve Center, assessed with the Stennert index (higher number = more improvement; absolute total Stennert index ranges from 0 to 10). The overall improvement of the complete study for the subgroup of acute versus chronic facial palsy as well as of several important subgroups with different therapy scenarios is shown. 

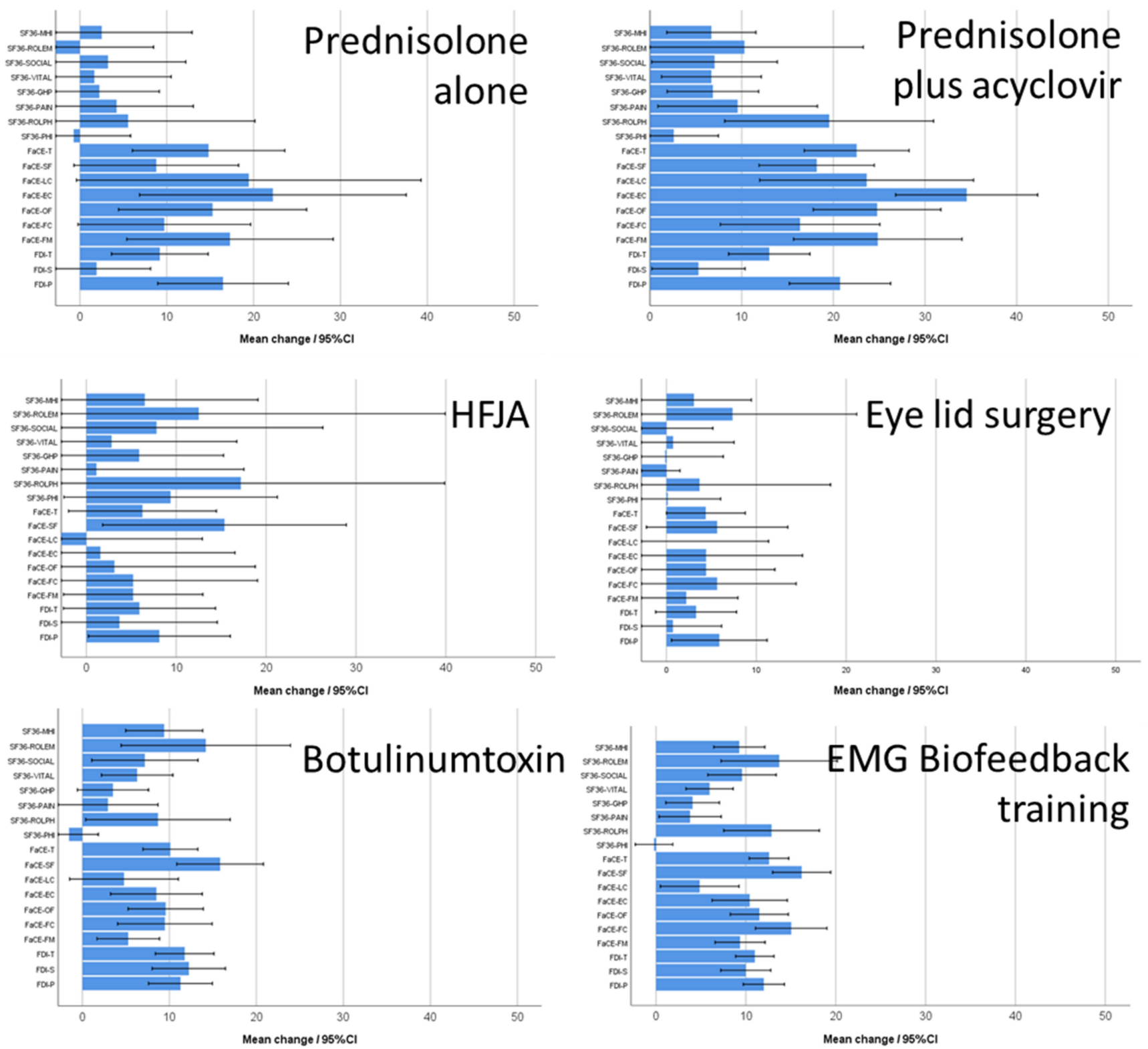

Figure 2. Absolute improvement of facial function between initial and last evaluation in the Facial Nerve Center, assessed with the Facial Disability Index (FDI), the Facial Clinimetric Evaluation Scale (FaCE), and 36-Item Short Form Survey (SF-36) separately for the same subgroups as in Figure 1. FDI-P = FDI physical function, FDI-S = FDI social function; FDI-T = FDI total; FaCE-FM = FaCE facial movement; FaCE-FC = FaCE facial comfort; FaCE-OF = FaCE oral function; FaCE-EC = FaCE eye comfort; FaCE-LC = FaCE lacrimal control; FaCE-SF $=$ FaCE social function; FaCE-T = FaCE total score; SF36-PHI = = SF-36 physical functioning; SF36-ROLPH = SF-36 physical role functioning; SF36-PAIN = SF-36 bodily pain; SF36-GHP = general health perceptions; SF36-VITAL = SF-36 vitality; SF36-SOCIAL = SF-36 social role functioning; SF36-ROLEM = SF-36 emotional role functioning; SF36-MHI = SF-36 mental health. More details on other treatment types are shown in Figure S4.

\section{Discussion}

Data on the outcome of treatment for acute or chronic facial palsy based on large series from multidisciplinary facial nerve centers are sparse. Specifically, large series reporting standardized outcome measures of a treatment in large-volume facial nerve centers are lacking. To our knowledge, the series from the facial nerve center in the Massachusetts Eye and Ear Infirmary, Boston, with 1989 patients treated between 2003 and 2013, is, so 
far, the largest [4]. The series from Boston presents patient and treatment characteristics as well as algorithms for decision-making. Outcome measures are not presented in this series. This also applies to the recent series of the facial nerve unit in Madrid [5]. The recent series from the Sydney Facial Nerve Clinic is much smaller, analyzing 145 patients treated between 2015 and 2018 [10], but it reports, at least, facial grading and facial-specific PROM data at initial referral. Krane et al. from Oregon focused on the surgical reanimation of 22 patients when reporting about the implementation of a facial nerve center with initial data from 2014 and 2019 [9]. They point out how important it is to measure outcomes when using a multidisciplinary approach to build up a facial nerve center. They used quantitative data (FACE-gram and Emotrics) as well as PROMs (FaCE and the Synkinesis Assessment Questionnaire (SAQ)) [34-36]. Moreover, a recent meta-analysis has shown that facial grading only explains a small part of the patient's quality of life; the association between grading and facial PROMs is low to moderate [37]. The present study is the first using a large and unselected series of a facial nerve center, beyond facial grading, with initial and follow-up FaCE, FDI, and SF-36 data. Confirming the results of other studies, facial-specific quality of life data (FaCE, FDI) is much more informative and meaningful than general quality of life data (SF-36) [38].

The number of studies measuring the outcome of a treatment for acute facial palsy with PROMs is small. Even the landmark phase III clinical trials of prednisolone treatment for Bell's palsy primarily relied on subjective facial grading [39,40]. In a small but prospective series of 21 patients with Bell's palsy, all FaCE subdomains significantly improved after corticosteroid treatment [41]. In a recent Swedish prospective trial of 96 patients with Bell's palsy, both the FDI and FaCE improved over time after corticosteroid treatment [42]. In these two studies, again, the correlation between facial grading and the PROM results was low to moderate. The present study could confirm these results in a large cohort of patients with Bell's palsy for a routine clinical setting in a specialized center but beyond clinical trials. PROM data for non-idiopathic acute palsy are given here for the first time.

The situation is very different for patients receiving facial nerve reconstruction surgery. Specifically, the facial nerve center in Boston (but also others) has published the outcome for selected cases, usually focused on a specific technique of reconstruction, based on PROMs such as the FDI and FaCE [33,43-48]. As in the present study, the effects of surgery are much better presented by PROM measures rather than facial grading. The same holds true for the treatment of synkinesis by, for instance, botulinum toxin treatment or other conservative measures $[49,50]$.

The present study has several limitations. The retrospective analysis can suggest associations but cannot analyze any causality. Although decision-making for diagnostics and treatment follows general rules in the center, the individual decision or deviation from standards could not be analyzed. All patients were treated. Hence, we cannot report on the spontaneous improvement or deterioration of the disease. In contrast to prospective trials, the facial grading and PROM measurements were not performed at defined follow-up times. To overcome this limitation, the Kaplan-Meier method was used to include time as a variable when measuring the outcome after treatment of acute facial palsy. Concerning outcomes for the treatment of chronic facial palsy, a sufficient follow-up time was ensured: $74 \%$ and $64 \%$ of the chronic facial palsy group had a follow-up of longer than 4 and 6 months, respectively. As is typical for the treatment of chronic facial palsy, most patients received combinations of different treatments (several types of surgery, combination of surgical with non-surgical treatment). Therefore, it was not feasible to perform a multivariable analysis after the univariable analysis (focus on treatment). The subgroups of identical treatment combinations were too small to allow multivariable analysis. Therefore, we could not analyze the effect of the interaction between the different treatment types on the outcome in patients with chronic facial palsy. With 95 cases, children were a minority; hence, they may be underrepresented. Patients with congenital lesions and candidates for free flap reconstruction were underrepresented as well. The evolution within the last few years show that the referral of such cases will increase in the future. Other 
facial nerve centers, including ours, have focused on the standardization of diagnostics, treatment, and outcome measures $[4,35]$. The next step will be the introduction of objective, automated, therapist-independent measurement tools to evaluate the outcome. After a decade of research, the first automated tools feasible for use in clinical routine have been published [51,52]. Furthermore, the referral to comprehensive facial palsy services still needs to be improved by the continuous training of our colleagues. The rate of treated cases of Bell's palsy without recovery, referred to secondary care, still seems to be too low; hence, many patients with synkinetic recovery remain untreated [2]. In the present study, $44 \%$ and $39 \%$ of the chronic cases were referred not before 12 and 9 months, respectively, after onset.

\section{Conclusions}

In this large and unselected series of patients with acute facial palsy, chronic flaccid paralysis, or post-paralytic synkinesis, it has been shown that a standardized approach in a multidisciplinary and inter-professional facial nerve center leads to the improvement of facial function and the related quality of life. To reach satisfactory results, a fast referral of complex acute cases, if recovery of normal facial function is unlikely or definitive, is recommended. Timely referral is very important. The center has to guarantee a large spectrum of up-to-date diagnostics, surgical techniques, and conservative measures to decide the best solution in individual cases.

Supplementary Materials: The following supporting information can be downloaded at: https:// www.mdpi.com/article/10.3390/jcm11020427/s1, Figure S1: 1212 of the 1220 patients were referred from all over Germany. Eight patients were referred from abroad. The Facial Nerve Center is located in Jena (red spot), a town in the federal state Thuringia. The frequency table in the lower right corner shows a continuously increasing referral from 2007 to 2018; Figure S2: Patients' facial nerve function at first and last presentation in the Facial Nerve center; Figure S3: Probability of complete recovery from acute facial palsy related to: A: initial Stennert index (SI); B: initial Facial Clinimetric Evaluation Scale total score (FaCE-T); C: Stapedius reflex test result; D: application of combined prednisolone and acyclovir treatment; E: the etiology. P-values of log-rank tests indicated; Figure S4: More detailed version of Figure 2: Absolute improvement of facial function between initial and last evaluation in the Facial Nerve Center, assessed with the Facial Disability Index (FDI), the Facial Clinimetric Evaluation Scale (FaCE), and 36-Item Short Form Survey (SF-36) for the entire study group and separately for subgroups of different treatment types; Table S1: Patients' characteristics ( $\mathrm{N}=1220)$; Table S2: Overview about absolute number of diagnostics $(\mathrm{N}=1220)$ and frequency per patient; Table S3: Treatment of all patients $(\mathrm{N}=1220)$; Table S4: Facial nerve function at initial and last visit $(\mathrm{N}=$ 1220); Table S5: Comparison of outcome measures between first and last visit *; Table S6: Comparison of outcome measures between first and last visit *; Table S7: Comparison of outcome measures between first and last visit *; Table S8: Association between patients' / treatment characteristics and probability of recovery after acute facial palsy $(\mathrm{N}=690 *)$; Table S9: Multivariable Cox regression of prognostic factors for recovery from acute facial palsy $(\mathrm{N}=690 *$ ).

Author Contributions: Conceptualization, O.G.-L., G.F.V. and C.D.; methodology, O.G.-L., G.F.V., C.M.K. and C.D.; formal analysis, J.S., O.G.-L. and C.D.; investigation, J.S., G.F.V., J.T., M.G. and A.-M.K.; resources, O.G.-L., C.M.K. and C.D.; writing—original draft preparation, O.G.-L.; writing—review and editing, all authors; visualization, O.G.-L. All authors have read and agreed to the published version of the manuscript.

Funding: Orlando Guntinas-Lichius acknowledges support by a Deutsche Forschungsgemeinschaft (DFG) grant GU-463/12-1.

Institutional Review Board Statement: The study was conducted in accordance with the Declaration of Helsinki, and approved by the Ethics Committee of Jena University Hospital, Jena, Germany (protocol code No. 2019-1539_6-BO, October 2019). for studies involving humans.

Informed Consent Statement: Informed consent was obtained from all subjects involved in the study.

Data Availability Statement: The datasets used during the current study are available from the corresponding. 
Acknowledgments: We thank the whole team of the Facial Nerve Center, Jena University Hospital, Germany. We especially thank Eva Miltner and Hendrik Möbius for their engaged assessments and the training of the patients with post-paralytic facial synkinesis in the multidisciplinary center.

Conflicts of Interest: The authors declare no conflict of interest.

Statement of Financial Interest: The authors have no financial interest to declare in relation to the content of this article.

\section{References}

1. $\quad$ Butler, D.P.; Morales, D.R.; Johnson, K.; Nduka, C. Facial palsy: When and why to refer for specialist care. Br. J. Gen. Pract. J. R. Coll. Gen. Pract. 2019, 69, 579-580. [CrossRef] [PubMed]

2. Morales, D.R.; Donnan, P.T.; Daly, F.; Staa, T.V.; Sullivan, F.M. Impact of clinical trial findings on Bell's palsy management in general practice in the UK 2001-2012: Interrupted time series regression analysis. BMJ Open 2013, 3, e003121. [CrossRef]

3. Plumbaum, K.; Volk, G.F.; Boeger, D.; Buentzel, J.; Esser, D.; Steinbrecher, A.; Hoffmann, K.; Jecker, P.; Mueller, A.; Radtke, G.; et al. Inpatient treatment of patients with acute idiopathic peripheral facial palsy: A population-based healthcare research study. Clin. Otolaryngol. 2017, 42, 1267-1274. [CrossRef]

4. Hohman, M.H.; Hadlock, T.A. Etiology, diagnosis, and management of facial palsy: 2000 patients at a facial nerve center. Laryngoscope 2014, 124, E283-E293. [CrossRef] [PubMed]

5. $\quad$ Lassaletta, L.; Morales-Puebla, J.M.; Gonzalez-Otero, T.; Moraleda, S.; Roda, J.M.; Gavilan, J. The Experience of a Facial Nerve Unit in the Treatment of Patients with Facial Paralysis Following Skull Base Surgery. Otol. Neurotol. 2020, 41, e1340-e1349. [CrossRef]

6. Glass, G.E.; Tzafetta, K. Optimising treatment of Bell's Palsy in primary care: The need for early appropriate referral. Br. J. Gen. Pract. J. R. Coll. Gen. Pract. 2014, 64, e807-e809. [CrossRef] [PubMed]

7. Butler, D.P.; Grobbelaar, A.O. Facial palsy: What can the multidisciplinary team do? J. Multidiscip. Healthc. 2017, 10, 377-381 [CrossRef]

8. Eviston, T.J.; Croxson, G.R.; Kennedy, P.G.; Hadlock, T.; Krishnan, A.V. Bell's palsy: Aetiology, clinical features and multidisciplinary care. J. Neurol. Neurosurg. Psychiatry 2015, 86, 1356-1361. [CrossRef] [PubMed]

9. Krane, N.A.; Chen, J.S.; Hanseler, H.; Baghoomian, W.; Ng, J.; Wax, M.K.; Loyo, M. Early Outcomes in an Emerging Facial Nerve Center: The Oregon Health and Science University (OHSU) Experience. Ann. Otol. Rhinol. Laryngol. 2021, 130, 459-466. [CrossRef]

10. Hayler, R.; Clark, J.; Croxson, G.; Coulson, S.; Hussain, G.; Ngo, Q.; Ch'ng, S.; Low, T.H. Sydney Facial Nerve Clinic: Experience of a multidisciplinary team. ANZ J. Surg. 2020, 90, 856-860. [CrossRef]

11. Adour, K.K.; Byl, F.M.; Hilsinger, R.L., Jr.; Kahn, Z.M.; Sheldon, M.I. The true nature of Bell's palsy: Analysis of 1000 consecutive patients. Laryngoscope 1978, 88, 787-801. [CrossRef]

12. Devriese, P.P.; Schumacher, T.; Scheide, A.; de Jongh, R.H.; Houtkooper, J.M. Incidence, prognosis and recovery of Bell's palsy. A survey of about 1000 patients (1974-1983). Clin. Otolaryngol. Allied Sci. 1990, 15, 15-27. [CrossRef]

13. Peitersen, E. Bell's palsy: The spontaneous course of 2500 peripheral facial nerve palsies of different etiologies. Acta Otolaryngol. 2002, 122, 4-30. [CrossRef]

14. Urban, E.; Volk, G.F.; Geissler, K.; Thielker, J.; Dittberner, A.; Klingner, C.; Witte, O.W.; Guntinas-Lichius, O. Prognostic factors for the outcome of Bells' palsy: A cohort register-based study. Clin. Otolaryngol. 2020, 45, 754-761. [CrossRef] [PubMed]

15. Geissler, K.; Urban, E.; Volk, G.F.; Klingner, C.M.; Witte, O.W.; Guntinas-Lichius, O. Non-idiopathic peripheral facial palsy: Prognostic factors for outcome. Eur. Arch. Oto-Rhino-Laryngol. 2021, 278, 3227-3235. [CrossRef]

16. Guntinas-Lichius, O.; Volk, G.F.; Olsen, K.D.; Makitie, A.A.; Silver, C.E.; Zafereo, M.E.; Rinaldo, A.; Randolph, G.W.; Simo, R.; Shaha, A.R.; et al. Facial nerve electrodiagnostics for patients with facial palsy: A clinical practice guideline. Eur. Arch. Oto-Rhino-Laryngol. 2020, 277, 1855-1874. [CrossRef] [PubMed]

17. Volk, G.F.; Leier, C.; Guntinas-Lichius, O. Correlation between electromyography and quantitative ultrasonography of facial muscles in patients with facial palsy. Muscle Nerve 2016, 53, 755-761. [CrossRef] [PubMed]

18. Puls, W.C.; Jarvis, J.C.; Ruck, A.; Lehmann, T.; Guntinas-Lichius, O.; Volk, G.F. Surface electrical stimulation for facial paralysis is not harmful. Muscle Nerve 2020, 61, 347-353. [CrossRef] [PubMed]

19. Arnold, D.; Thielker, J.; Klingner, C.M.; Puls, W.C.; Misikire, W.; Guntinas-Lichius, O.; Volk, G.F. Selective Surface Electrostimulation of the Denervated Zygomaticus Muscle. Diagnostics 2021, 11, 188. [CrossRef] [PubMed]

20. Stennert, E.; Limberg, C.H.; Frentrup, K.P. An index for paresis and defective healing-an easily applied method for objectively determining therapeutic results in facial paresis (author's transl). HNO 1977, 25, 238-245. [PubMed]

21. VanSwearingen, J.M.; Brach, J.S. Validation of a treatment-based classification system for individuals with facial neuromotor disorders. Phys. Ther. 1998, 78, 678-689. [CrossRef] [PubMed]

22. Kahn, J.B.; Gliklich, R.E.; Boyev, K.P.; Stewart, M.G.; Metson, R.B.; McKenna, M.J. Validation of a patient-graded instrument for facial nerve paralysis: The FaCE scale. Laryngoscope 2001, 111, 387-398. [CrossRef]

23. Volk, G.F.; Steigerwald, F.; Vitek, P.; Finkensieper, M.; Kreysa, H.; Guntinas-Lichius, O. Facial Disability Index and Facial Clinimetric Evaluation Scale: Validation of the German versions. Laryngo-Rhino-Otol. 2015, 94, 163-168. [CrossRef] 
24. Ware, J.E., Jr.; Gandek, B. Overview of the SF-36 Health Survey and the International Quality of Life Assessment (IQOLA) Project. J. Clin. Epidemiol. 1998, 51, 903-912. [CrossRef]

25. Phillips, K.A. Understanding Body Dysmorphic Disorder; Oxford University Press: New York, NY, USA, 2009.

26. Beck, A.T.; Ward, C.H.; Mendelson, M.; Mock, J.; Erbaugh, J. An inventory for measuring depression. Arch. Gen. Psychiatry 1961, 4, 561-571. [CrossRef]

27. Liebowitz, M.R. Anxiety. Mod. Trends Pharm. 1987, 22, 141-173.

28. Volk, G.F.; Hesse, S.; Geissler, K.; Kuttenreich, A.M.; Thielker, J.; Dobel, C.; Guntinas-Lichius, O. Role of Body Dysmorphic Disorder in Patients with Postparalytic Facial Synkinesis. Laryngoscope 2021, 131, E2518-E2524. [CrossRef] [PubMed]

29. Heckmann, J.G.; Lang, C.; Urban, P.; Glocker, F.X.; Weder, B.; Reiter, G.; Bischoff, C.; Meier, U.; Guntinas-Lichius, O. Treatment for Idiopathic Facial Nerve Palsy (Bell's palsy). Akt. Neurol. 2017, 44, 712-727, epub ahead of print.

30. Heckmann, J.G.; Urban, P.P.; Pitz, S.; Guntinas-Lichius, O.; Gagyor, I. The Diagnosis and Treatment of Idiopathic Facial Paresis (Bell's Palsy). Dtsch. Arztebl. Int. 2019, 116, 692-702. [CrossRef]

31. Guntinas-Lichius, O.; Streppel, M.; Stennert, E. Postoperative functional evaluation of different reanimation techniques for facial nerve repair. Am. J. Surg. 2006, 191, 61-67. [CrossRef] [PubMed]

32. Volk, G.F.; Pantel, M.; Guntinas-Lichius, O. Modern concepts in facial nerve reconstruction. Head Face Med. 2011, 6, 25. [CrossRef]

33. Volk, G.F.; Geitner, M.; Geissler, K.; Thielker, J.; Raslan, A.; Mothes, O.; Dobel, C.; Guntinas-Lichius, O. Functional Outcome and Quality of Life After Hypoglossal-Facial Jump Nerve Suture. Front. Surg. 2020, 7, 11. [CrossRef]

34. Mehta, R.P.; WernickRobinson, M.; Hadlock, T.A. Validation of the Synkinesis Assessment Questionnaire. Laryngoscope 2007, 117, 923-926. [CrossRef]

35. Hadlock, T.A.; Urban, L.S. Toward a universal, automated facial measurement tool in facial reanimation. Arch. Facial Plast. Surg. 2012, 14, 277-282. [CrossRef] [PubMed]

36. Guarin, D.L.; Dusseldorp, J.; Hadlock, T.A.; Jowett, N. A Machine Learning Approach for Automated Facial Measurements in Facial Palsy. JAMA Facial Plast. Surg. 2018, 20. [CrossRef]

37. Bruins, T.E.; van Veen, M.M.; Werker, P.M.N.; Dijkstra, P.U.; Broekstra, D.C. Associations Between Clinician-Graded Facial Function and Patient-Reported Quality of Life in Adults with Peripheral Facial Palsy: A Systematic Review and Meta-analysis. JAMA Otolaryngol.—Head Neck Surg. 2021, 147, 717-728. [CrossRef] [PubMed]

38. Volk, G.F.; Granitzka, T.; Kreysa, H.; Klingner, C.M.; Guntinas-Lichius, O. Nonmotor disabilities in patients with facial palsy measured by patient-reported outcome measures. Laryngoscope 2016, 126, 1516-1523. [CrossRef] [PubMed]

39. Sullivan, F.M.; Swan, I.R.; Donnan, P.T.; Morrison, J.M.; Smith, B.H.; McKinstry, B.; Davenport, R.J.; Vale, L.D.; Clarkson, J.E.; Hammersley, V.; et al. Early treatment with prednisolone or acyclovir in Bell's palsy. N. Engl. J. Med. 2007, 357, 1598-1607. [CrossRef] [PubMed]

40. Engstrom, M.; Berg, T.; Stjernquist-Desatnik, A.; Axelsson, S.; Pitkaranta, A.; Hultcrantz, M.; Kanerva, M.; Hanner, P.; Jonsson, L. Prednisolone and valaciclovir in Bell's palsy: A randomised, double-blind, placebo-controlled, multicentre trial. Lancet Neurol. 2008, 7, 993-1000. [CrossRef]

41. Ng, J.H.; Ngo, R.Y. The use of the facial clinimetric evaluation scale as a patient-based grading system in Bell's palsy. Laryngoscope 2013, 123, 1256-1260. [CrossRef]

42. Bylund, N.; Hultcrantz, M.; Jonsson, L.; Marsk, E. Quality of Life in Bell's Palsy: Correlation with Sunnybrook and HouseBrackmann Over Time. Laryngoscope 2021, 131, E612-E618. [CrossRef]

43. Lindsay, R.W.; Bhama, P.; Hadlock, T.A. Quality-of-life improvement after free gracilis muscle transfer for smile restoration in patients with facial paralysis. JAMA Facial Plast. Surg. 2014, 16, 419-424. [CrossRef] [PubMed]

44. Bhama, P.K.; Park, J.G.; Shanley, K.; Hadlock, T.A. Refinements in nasolabial fold reconstruction for facial paralysis. Laryngoscope 2014, 124, 2687-2692. [CrossRef]

45. Pavese, C.; Cecini, M.; Lozza, A.; Biglioli, F.; Lisi, C.; Bejor, M.; Dalla Toffola, E. Rehabilitation and functional recovery after masseteric-facial nerve anastomosis. Eur. J. Phys. Rehabil. Med. 2016, 52, 379-388.

46. Dusseldorp, J.R.; van Veen, M.M.; Mohan, S.; Hadlock, T.A. Outcome Tracking in Facial Palsy. Otolaryngol. Clin. N. Am. 2018, 51, 1033-1050. [CrossRef]

47. Greene, J.J.; Tavares, J.; Mohan, S.; Jowett, N.; Hadlock, T. Long-Term Outcomes of Free Gracilis Muscle Transfer for Smile Reanimation in Children. J. Pediatrics 2018, 202, 279-284.e272. [CrossRef] [PubMed]

48. Parrino, D.; Franchella, S.; Frigo, A.C.; Mazzoni, A.; Marioni, G.; Zanoletti, E. Facial nerve sacrifice in lateral approaches to the skull base: Simultaneous reconstruction by graft interposition. Am. J. Otolaryngol. 2021, 43, 103210. [CrossRef] [PubMed]

49. Mehta, R.P.; Hadlock, T.A. Botulinum toxin and quality of life in patients with facial paralysis. Arch. Facial Plast. Surg. 2008, 10, 84-87. [CrossRef]

50. van Veen, M.M.; Tavares-Brito, J.; van Veen, B.M.; Dusseldorp, J.R.; Werker, P.M.N.; Dijkstra, P.U.; Hadlock, T.A. Association of Regional Facial Dysfunction with Facial Palsy-Related Quality of Life. JAMA Facial Plast. Surg. 2019, 21, 32-37. [CrossRef]

51. Mothes, O.; Modersohn, L.; Volk, G.F.; Klingner, C.; Witte, O.W.; Schlattmann, P.; Denzler, J.; Guntinas-Lichius, O. Automated objective and marker-free facial grading using photographs of patients with facial palsy. Eur. Arch. Oto-Rhino-Laryngol. 2019, 276, 3335-3343. [CrossRef]

52. Miller, M.Q.; Hadlock, T.A.; Fortier, E.; Guarin, D.L. The Auto-eFACE: Machine Learning-Enhanced Program Yields Automated Facial Palsy Assessment Tool. Plast. Reconstr. Surg. 2021, 147, 467-474. [CrossRef] [PubMed] 